\title{
Problems on Robin Inequality and Riemann Hypothesis
}

\author{
Jamal Salah \\ Department of Basic Science, College of Health and Applied Sciences \\ A'Sharqiyah University, Ibra post code 400 Oman \\ damous73@yahoo.com
}

\begin{abstract}
In this piece of work we explore the sum of divisors functions as coefficient in a series of real (complex) numbers. We provide a brief conclusion and evoke the connection to Robin inequality which is equivalent to Riemann Hypothesis.
\end{abstract}

MSC 2010 Classification: 97I80, 11M41, 11A41

Keywords: Robin Inequality, Riemann Hypothesis, Analytic Continuity, non-trivial zeta zeros

Robin Inequality (See [1] and [2]) Riemann Hypothesis is equivalent to

$$
\begin{gathered}
\sigma(n)<n e^{\gamma} \ln \ln n, \quad n>5040, \quad \gamma=0.57721 \ldots \\
\sigma(n): \text { The sum of divisors function }
\end{gathered}
$$

The one can readily deduce the following equation

$$
\sum_{n=1}^{\infty} \frac{z^{n}}{\left(1-z^{n}\right)^{2}}=\sum_{n=1}^{\infty} \sigma(n) z^{n}
$$

Where $z$ real (complex)number, The LHS converges everywhere except at $z=1$ 


\section{Problem I:}

Write

$$
\sum_{n=1}^{\infty} \frac{z^{n}}{\left(1-z^{n}\right)^{2}}=\sum_{n=1}^{\infty} f(n) z^{n}
$$

Find $\epsilon_{n}$ such that

$$
|\sigma(n)-f(n)|<\epsilon_{n}, \quad n=5041,5042, \ldots
$$

Then prove that:

$$
\sigma(n)<f(n)+\epsilon_{n}<e^{\gamma} n \ln \ln n, \quad n=5041,5042, \ldots
$$

Or

$$
\sigma(n)<f(n)+\epsilon<e^{\gamma} n \ln \ln n, \quad \epsilon=\min \left\{\epsilon_{n}, \quad n=5041,5042, \ldots\right\}
$$

\section{Problem II:}

From (1) and for a fixed $N>5041$

$$
\sigma(N)=\sum_{n=1}^{\infty} \frac{z^{n-N}}{\left(1-z^{n}\right)^{2}}-\sum_{\substack{n=1 \\ n \neq N}}^{\infty} \sigma(n) z^{n-N}
$$

Show that:

$$
\sum_{n=1}^{\infty} \frac{z^{n-N}}{\left(1-z^{n}\right)^{2}}-\sum_{\substack{n=1 \\ n \neq N}}^{\infty} \sigma(n) z^{n-N}<e^{\gamma} N \ln \ln N
$$




\section{References}

[1] Salah, Jamal Y. "On Riemann hypothesis and Robin's inequality." International Journal of Scientific and Innovative Mathematical Research (IJSIMR) 3.4 (2015): 9-14.

[2] Young-Ju Choie, Nicolas Lichiardopol, Pieter Moree and Patrick Solé, On Robin's criterion for Riemann Hypothesis, Journal de Théor. Nombres. Bordeaux 19 (2007), no 2, $357-372$. 